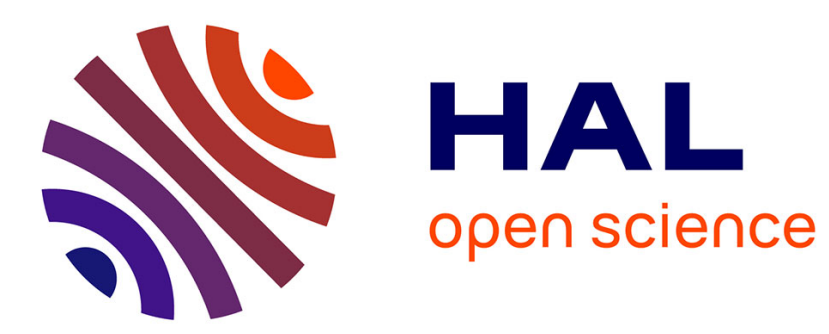

\title{
Multiagent Approach for the Representation of Information in a Decision Support System
}

\author{
Fahem Kebair, Frédéric Serin
}

\section{To cite this version:}

Fahem Kebair, Frédéric Serin. Multiagent Approach for the Representation of Information in a Decision Support System. SpringerLink. Springer Berlin / Heidelberg, pp.98-107, 2006, Lecture Notes in Computer Science. hal-00266463

\section{HAL Id: hal-00266463 https://hal.science/hal-00266463}

Submitted on 24 Mar 2008

HAL is a multi-disciplinary open access archive for the deposit and dissemination of scientific research documents, whether they are published or not. The documents may come from teaching and research institutions in France or abroad, or from public or private research centers.
L'archive ouverte pluridisciplinaire HAL, est destinée au dépôt et à la diffusion de documents scientifiques de niveau recherche, publiés ou non, émanant des établissements d'enseignement et de recherche français ou étrangers, des laboratoires publics ou privés. 


\title{
Multiagent Approach for the Representation of Information in a Decision Support System
}

\author{
Fahem Kebair and Frédéric Serin \\ Université du Havre, LITIS - Laboratoire d'Informatique, \\ de Traitement de l'Information et des Systèmes, \\ 25 rue Philippe Lebon, 76058, Le Havre Cedex, France \\ \{fahem.kebair, frederic.serin\}@univ-lehavre.fr
}

\begin{abstract}
In an emergency situation, the actors need an assistance allowing them to react swiftly and efficiently. In this prospect, we present in this paper a decision support system that aims to prepare actors in a crisis situation thanks to a decision-making support. The global architecture of this system is presented in the first part. Then we focus on a part of this system which is designed to represent the information of the current situation. This part is composed of a multiagent system that is made of factual agents. Each agent carries a semantic feature and aims to represent a partial part of a situation. The agents develop thanks to their interactions by comparing their semantic features using proximity measures and according to specific ontologies.
\end{abstract}

Keywords. Decision support system, Factual agent, Indicators, Multiagent system, Proximity measure, Semantic feature.

\section{Introduction}

Making a decision in a crisis situation is a complicated task. This is mainly due to the unpredictability and the rapid evolution of the environment state. Indeed, in a critic situation time and resources are limited. Our knowledge about the environment is incomplete and uncertain, verily obsolete. Consequently, it is difficult to act and to adapt to the hostile conditions of the world. This makes sense to the serious need of robust, dynamic and intelligent planning system for search-and-rescue operations to cope with the changing situation and to best save people [9]. The role of such a system is to provide an emergency planning that allows actors to react swiftly and efficiently to a crisis case.

In this context, our aim is to build a system designed to help decision-makers manage cases of crisis with an original representation of information. From the system point of view, detecting a crisis implies its representation, its characterisation and its comparison permanently with other crisis stored in scenarios base. The result of this comparison is provided to the user as the answer of the global system .

The idea began with the speech interpretation of human actors during a crisis [3], [5]. The goal was to build an information, and communication system (ICS) 
which enables the management of emergency situations by interpreting aspects communications created by the actors. Then, a preventive vigil system (PVS) [1] was designed with the mean of some technologies used in the ICS modelling as: semantic features, ontologies, and agents with internal variables and behavioural automata. The PVS aims either to prevent a crisis or to deal with it with a main internal goal: detecting a crisis.

Since 2003, the architecture of the PVS was redesigned with a new specificity, that is the generic aspect; generic is used here with different meaning from [13]. A part of the global system, which is responsible of the dynamic information representation of the current situation, was applied to the game of Risk and tested thanks to a prototype implemented in Java [10]. However, we postulate that some parts of the architecture and, at a deeper level, some parts of the agents were independent of the subject used as application. Therefore, the objective at present is to connect this part to the other parts, that we present latter in this paper, and to test the whole system on various domains, as RoboCup Rescue [11] and e-learning.

We focus here on the modelling of the information representation part of the system that we intend to use it in a crisis management support system.

The paper begins with the presentation of the global system architecture. The core of the system is constituted by a multiagent system (MAS) which is structured on three multiagent layers. Then, in section 3 , we explain the way we formalise the environment state and we extract information related to it, which are written in the form of semantic features. The latter constitute data that feed the system permanently and that carry information about the current situation. The semantic features are handled by factual agents and are compared the one with the other using specific ontologies [2].

Factual agents, that compose the first layer of the core, are presented thereafter in section 4 . Each agent carries a semantic feature and aims to reflect a partial part of the situation. We present their structures and their behaviours inside their organisation using internal automaton and indicators.

Finally, we present a short view about the game of Risk test in which we describe the model application and the behaviour of factual agents.

\section{Architecture of the Decision Support System}

The role of the decision support system (DSS) is to provide a decision-making support to the actors in order to assist them during a crisis case. The DSS allows also managers to anticipate the occur of potential incidents thanks to a dynamic and a continuous evaluation of the current situation. Evaluation is realised by comparing the current situation with past situations stored in a scenarios base. The latter can be viewed as one part of the knowledge we have on the specific domain.

The DSS is composed of a core and three parts which are connected to it (figure 1): 
- A set of user-computer interfaces and an intelligent interface allow the core to communicate with the environment. The intelligent interface controls and manages the access to the core of the authenticated users, filters entries information and provides actors with results emitted by the system;

- An inside query $M A S$ ensures the interaction between the core and world information. These information represent the knowledge the core need. The knowledge includes the scenarios, that are stored in a scenarios base, the ontologies of the domain and the proximity measures;

- An outside query MAS has as role to provide the core with information, that are stored in network distributed information systems.

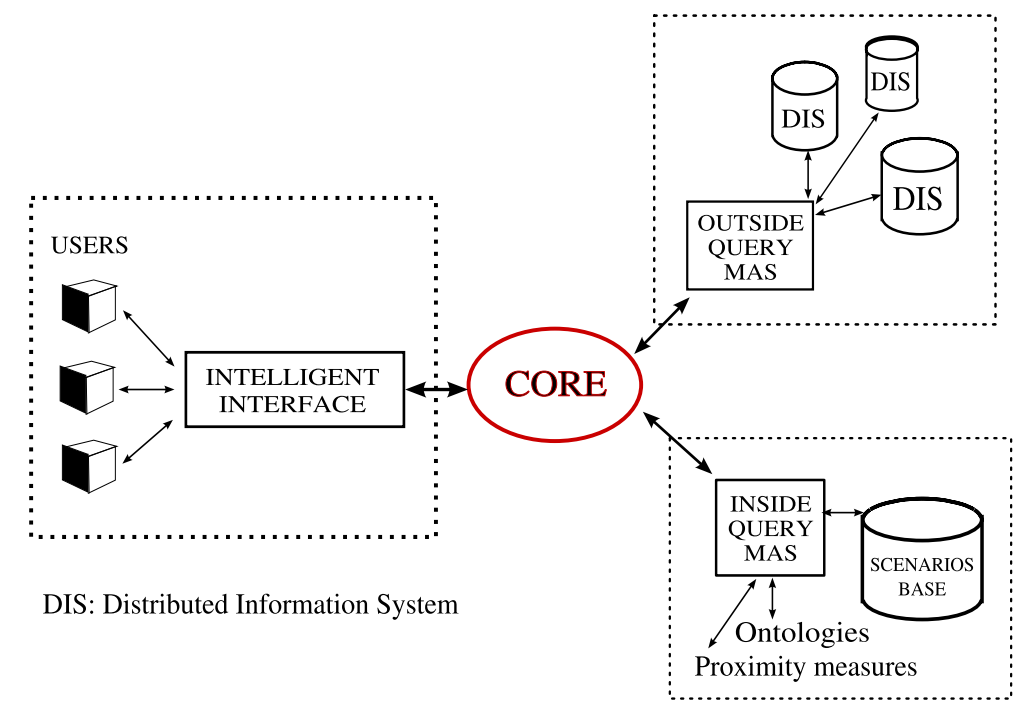

Fig. 1. General Architecture of the DSS

The core of the decision support system is made of a MAS which is structured on three layers. The latter contain specific agents that differ in their objectives and their communications way. In a first time, the system describes the semantic of the current situation thanks to data collected from the environment. Then it analyses pertinent information extracted from the scenario. Finally, it provides an evaluation of the current situation and a decision support using a dynamic and incremental case-base reasoning.

The three layers of the core are:

- The lowest layer: factual agents;

- The intermediate layer: synthesis agents;

- The highest layer: prediction agents. 
Information are coming from the environment in the form of semantic features without a priori knowledge of their importance. The role of the first layer (the lowest one) is to deal with these data thanks to factual agents and let emergence detect some subsets of all the information [7]. More precisely, the set of these agents will enable the appearance of a global behaviour thanks to their interactions and their individual operations. The system will extract thereafter from this behaviour the pertinent information that represent the salient facts of the situation.

The role of the synthesis agents is to deal with the agents emerged from the first layer. Synthesis agents aim to create dynamically factual agents clusters according to their evolutions. Each cluster represents an observed scenario. The set of these scenarios will be compared to past ones in order to deduce their potential consequences.

Finally, the upper layer, will build a continuous and incremental process of recollection for dynamic situations. This layer is composed of prediction agents and has as goal to evaluate the degree of resemblance between the current situation and its associate scenario continuously. Each prediction agent will be associated to a scenario that will bring it closer, from semantic point of view, to other scenarios for which we know already the consequences. The result of this comparison constitutes a support information that can help a manager to make a good decision.

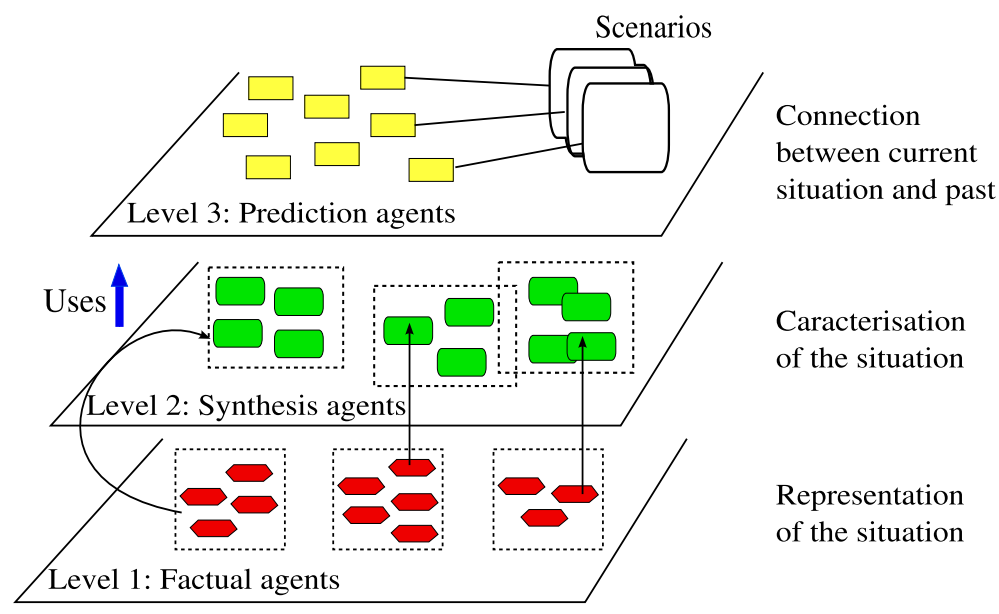

Fig. 2. Architecture of the Core 


\section{Environment Study and Creation of Semantic Features}

\subsection{Situation Formalisation}

To formalise a situation means to create a formal system, in an attempt to capture the essential features of the real-world. To realise this, we model the world as a collection of objects, where each one holds some properties. The aim is to define the environment objects following the object paradigm. Therefore, we build a structural and hierarchical form in order to give a meaning to the various relations that may exist between them. The dynamic change of these objects states and more still the interactions that could be entrenched between them will provide us a snapshot description of the environment. In our context, information are decomposed in atomic data where each one is associated to a given object.

\subsection{Semantic Features}

A semantic feature is an elementary piece of information coming from the environment and which represents a fact that occurred in the world. Each semantic feature is related to an object (defined in section 3.1), and allows to define all or a part of this object. A semantic feature has the following form: (key, $(\text { qualification, value })^{+}$), where key is the described object and (qualification, value $)^{+}$is a set of couples formed by: the qualification of the object and its associated value. As example of a semantic feature related to a phenomenon object: (phenomenon\#1, type, fire, location, \#4510, time, 9:33). The object described by this semantic feature is phenomenon\#1, and has as qualifications: type, location, and time.

The modelling of semantic features makes it possible to obtain a homogeneous structure. This homogeneity is of primary importance because it allows to establish comparisons between these data. The latter are managed by factual agents, where each one carries one semantic feature and of which behaviour depends on the type of this information.

According to FIPA communicative acts [6], the agents must share the same language and vocabulary to communicate. The use of semantic features in communications process implies to define an ontology.

Inside the representation layer (the first layer of the system), agents evolve by comparing their semantic features. These comparisons allow to establish semantic distances between the agents, and are computed thanks to proximity measures.

We distinguish three types of proximities: time proximity, spatial proximity and semantic proximity. The global proximity multiplies these three proximities together. The measurement of a semantic proximity is related to ontologies. Whereas time proximity and spatial proximity are computed according to specific functions.

Proximities computation provides values on $[-1,1]$ and is associated to a scale. The reference value in this scale is 0 that means neutral relation between 
the two compared semantic features. Otherwise, we can define the scale as follow: $0.4=$ Quiet Close, $0.7=$ Close, $0.9=$ Very Close, $1=$ Equal. Negative values mirrors positive ones (replacing close by different).

\section{Factual Agents}

\subsection{Presentation and Structure of a Factual Agent}

Factual agents are hybrid agents, they are both cognitive and reactive agents. They have therefore the following characteristics: reactivity, proactiveness and social ability [14]. Such an agent represents a feature with a semantic character and has also to formulate this character feature, a behaviour [4]. This behaviour ensures the agent activity, proactiveness and communication functions.

The role of a factual agent is to manage the semantic feature that it carries inside the MAS. The agent must develop to acquire a dominating place in its organisation and consequently, to make prevail the semantic category which it represents. For this, the factual agent is designed with an implicit goal that is to gather around it as much friends as possible in order to build a cluster. In other words, the purpose of the agent is to add permanently in its acquaintances network a great number of semantically close agents. The cluster formed by these agents is recognized by the system as a scenario of the current situation and for which it can bring a potential consequence. A cluster is formed only when its agents are enough strong and consequently they are in an advanced state in their automaton. Therefore, the goal of the factual agent is to reach the action state, in which is supreme and its information may be regarded by the system as relevant.

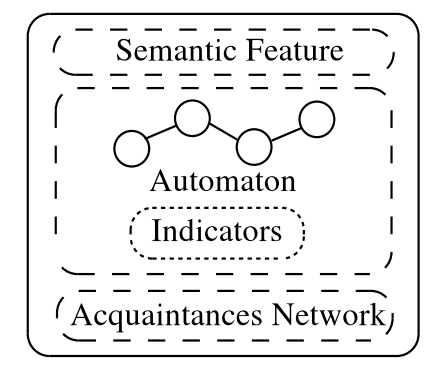

Fig. 3. Structure of a Factual Agent

An internal automaton describes the behaviour and defines the actions of the agent. Some indicators and an acquaintances network allow the automaton operation, that means they help the agent to progress inside its automaton and to execute actions in order to reach its goal. These characteristics express the proactiveness of the agent. 
The acquaintances network contains the addresses of the friends agents and the enemies agents used to send messages. This network is dynamically constructed and permanently updated. Agents are friends (enemies) if their semantic proximities are strictly positive (negative).

\subsection{Factual Agent Behaviour}

Behavioural Automaton The internal behaviour of a factual agent is described by a generic augmented transition network (ATN). The ATN is made of four states [3] (quoted above) linked by transitions:

- Initialisation state: the agent is created and enters in activities;

- Deliberation state: the agent searches in its acquaintances allies in order to achieve its goals;

- Decision state: the agent try to control its enemies to be reinforced;

- Action state: it is the state-goal of the factual agent, in which the latter demonstrates its strength by acting and liquidating its enemies.

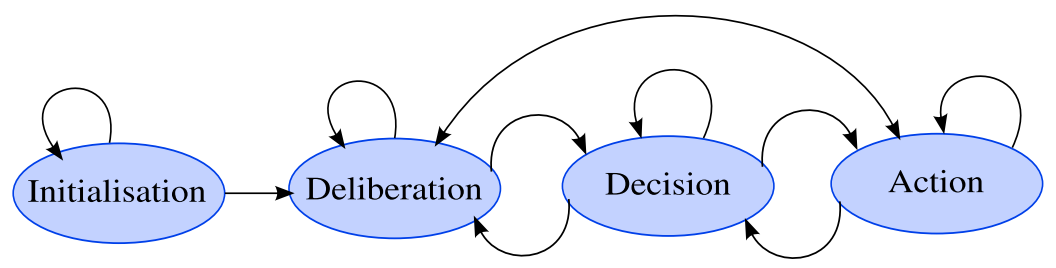

Fig. 4. Generic Automaton of a Factual Agent

ATN transitions are stamped by a set of conditions and a sequence of actions. Conditions are defined as thresholds using internal indicators. The agent must validate thus one of its outgoing current state transitions in order to pass to the next state. The actions of the agents may be an enemy aggression or a friend help. The choice of the actions to perform depend both on the type of the agent and its position in the ATN.

Factual Agent Indicators The dynamic measurement of an agent behaviour and its state progression at a given time are given thanks to indicators. These characters are significant parameters that describe the activities variations of each agent and its structural evolution. In other words, the agent state is specified by the set of these significant characters that allow both the description of its current situation and the prediction of its future behaviour [4] (quoted above).

Factual agent has five indicators, which are pseudoPosition (PP), pseudoSpeed (PS), pseudoAcceleration (PA), satisfactory indicator (SI) and constancy indicator (CI) [8]. The "pseudo" prefix means that these indicators are not a real 
mathematical speed or acceleration: we chose a constant interval of time of one between two evolutions of semantic features. PP represents the current position of an agent in the agent representation space. PS evaluates the PP evolution speed and PA means the PS evolution estimation. SI is a valuation of the success of a factual agent in reaching and staying in the deliberation state. This indicator measures the satisfaction degree of the agent. Whereas, CI represents the tendency of a given factual agent to transit both from a state to a different state and from a state to the same state. This allows the stability measurement of the agent behaviour.

The compute of these indicators is according to this formulae where valProximity depends on the category of a given application factual agents:

$$
\begin{aligned}
& P P_{t+1}=\text { valPoximity } \\
& P S_{t+1}=P P_{t+1}-P P_{t} \\
& P A_{t+1}=P S_{t+1}-P S_{t}
\end{aligned}
$$

PP, PS and PA represent thresholds that define the conditions of the ATN transitions. The definition of this conditions are specified to a given application. As shown in the previous formulae, only PP is specific. However, PS and PA are generic and are deduced from PP. SI and CI are also independent of the studied domain and are computed according to the agent movement in its ATN.

\section{Game of Risk Use Case}

The first layer model has been tested on the game of Risk. We chose this game as application not only because it is well suited for crisis management but also we apprehend the elements and the actions on such an environment. Moreover we have an expert [8] (quoted above) in our team who is able to evaluate and validate results at any moment.

As result, this test proved that this model allows the dynamic information representation of the current situation thanks to factual agents organisation. Moreover we could study the behaviour and the dynamic evolution of these agents.

Risk is a strategic game which is composed of a playing board representing a map of forty-two territories that are distributed on six continents. A player wins by conquering all territories or by completing his secret mission. In turn, each player receives and places new armies and may attack adjacent territories. An attack is one or more battles fought with dice. Rules, tricks and strategies are detailed in [12].

The representation layer of the system has as role to simulate the game unwinding and to provide a semantic instantaneous description of its current state. To achieve this task, we began by identifying the different objects that define the game board (figure 5) and which are: territory, player, army and continent. Continents and territories are regarded as descriptions of a persistent situation. Whereas, armies and players are activities respectively observed (occupying a territory) and driving the actions. 


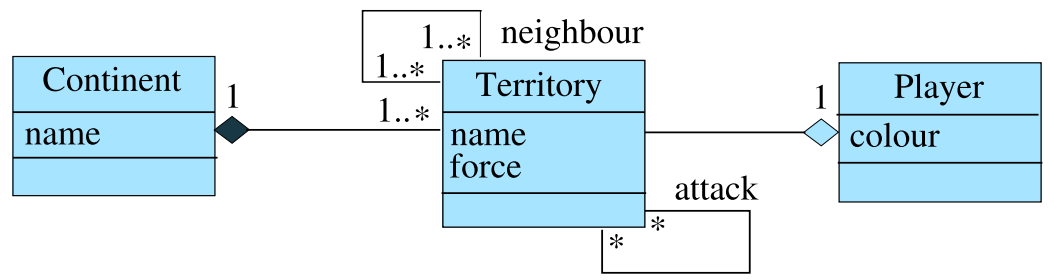

Fig. 5. Class Diagram for the Game of Risk Representation

From this model we distinguish two different types of semantic features: a player type and a territory type. For example (Quebec, player, green, nbArmies, 4 , time, 4) is a territory semantic feature that means Quebec territory is owned by the green player and has four armies. However, (blue, nbTerritories, 4, time, 1 ) is a player semantic feature that signifies a blue player has four territories at step 1.

The first extracted semantic features of the initial state of the game cause the creation of factual agents. For example, a semantic feature as (red, nbTerritories, 0 , time, 1) will cause the creation of red player factual agent.

During the game progression, the entry of a new semantic feature to the system may affect some agents state. A factual agent of type (Alaska, player, red, nbArmies, 3, time, 10) become (Alaska, player, red, nbArmies, -2, time, 49) with the entry of the semantic feature (Alaska, player, red, nbArmies, 1, time, 49). Alaska agent sends messages containing its semantic feature to all the other factual agents to inform them about its change. The other agents compare their own information with the received one. If an agent is interested by this message (the proximity measure between the two semantic features is not null) it updates its semantic feature accordingly. If the red player owned GB before the semantic feature (GB, player, blue, nbArmies, 5 , time, 52 ), both red player and blue player will receive messages because of the change of the territory owner.

If we take again the preceding example (Alaska territory), Alaska agent computes its new PP (valProximity). The computation of valProximity in our case is given by: number of armies ( $\mathrm{t}$ ) - number of armies ( $\mathrm{t}-1)$ e.g. here valProximity $=1-3=-2$. PS and PA are deduced thereafter from PP. The agent verify then the predicates of its current state outgoing transitions in order to change state. To pass from Deliberation state to Decision state for example the PS must be strictly positive. During this transition, the agent will send a SupportMessage to a friend and an AgressionMessage to an enemy.

\section{Conclusion}

The paper has presented a decision support system which aims to help decisionmakers to analyse and evaluate a current situation. The core of the system rests on an agent-oriented multilayer architecture. We have described here the first layer which aims to provide a dynamic information representation of the current 
situation and its evolution in time. This part is modelled with an original information representation methodology which is based on the handle of semantic features using a factual agents organisation.

The model of the first layer was applied on the game of Risk. Results provided by this test correspond to our attempts, which consist on the dynamic representation of information. This application allowed us to track the behaviour of factual agents and to understand their parameters which are the most accurate to characterise information. Moreover, we consider that a great part of the system is generic and may be carried into other fields. Currently, we intend in a first time to connect the representation layer to the two other and to apply thereafter the whole system on more significant domains as RoboCup Rescue and e-learning.

\section{References}

1. Boukachour, H.: Systéme de veille préventive pour la gestion de situations d'urgence: une modélisation par organisation d'agents. Application aux risques industriels. PhD Thesis, University of le Havre, France (2002)

2. Boukachour, H., Galinho, T., Person, P., Serin, F.: Towards an architecture for the representation of dynamic situations. In Las Vegas IC-AI 659-664 (2003)q

3. Cardon, A.: A multi-agent model for cooperative communications in crisis management system: the act of communication. In 7th European-Japanese Conference on Information Modeling and Knowledge-Based Simulation 111-123 (1997)

4. Cardon, A.: Modéliser et concevoir une machine pensante: approhce de la conscience artificielle, Vuibert, (2004)

5. Durand, S.: Représentation des points de vue multiples dans une situation d'urgnece: une modélisation par organisation d'agents. PhD Thesis, University of Le Havre, France (1999)

6. http://www.fipa.org, last retrieved March (2006)

7. Galinho, T., Serin, F.: Semantic Features And Factual Agents: A Model To Represent Changeable Situations A Model To Rpresent Changeable Situations (2003)

8. Galinho, T., Coletta, M., Person, P., Serin, F.: Dynamic Representation of Information for a Decision Support System, 8th International Conference on Entreprise Information, ICEIS2006, Paphos, Cyprus, (2006)

9. Kitano, H., Tadokoro, S.: RoboCup Rescue A Grand Challenge for Multiagent and Intelligent Systems, American Association for Artificial Intelligence (2001)

10. Person, P., Boukachour, H., Coletta, M., Galinho, T., Serin, F.: From Three MultiAgent Systems to One Decision Support System, $2^{\text {nd }}$ Indian International Conference on Artificial Intelligence, Pune, India (2005)

11. http://sasemas.org/2005/challenge.html, last retrieved March (2006) http://www.rescuesystem.org/robocuprescue, last retrieved March (2006)

12. http://www.thegamesjournal.com/articles/Risk.shtml, last retrieved March (2006)

http://www.tuxick.net/xfrisk/doc/riskfaq.html, last retrieved March (2006)

13. Wooldridge, M., Jennings, N.R.: Pitfalls of agent-oriented development $2^{\text {nd }}$ International Conference en Autonomous Agents, pp. 385-391, Minneapolis, (1998)

14. Wooldridge, M.: An Introduction to MultiAgent Systems, Wiley, (2002) 